\title{
Optical fiber sensors embedded in polymer flexible foils
}

\author{
Bram Van Hoe ${ }^{* a}$, Geert Van Steenberge ${ }^{\mathrm{a}}$, Erwin Bosman ${ }^{\mathrm{b}}$, Jeroen Missinne ${ }^{\mathrm{a}}$, Thomas Geernaert ${ }^{\mathrm{c}}$, \\ Francis Berghmans ${ }^{c}$, David Webb ${ }^{\mathrm{d}}$, Peter Van Daele ${ }^{\mathrm{b}}$ \\ ${ }^{a}$ Centre for Microsystems Technology (CMST), Elis Department, Ghent University-imec, Belgium \\ ${ }^{\mathrm{b}}$ Centre for Microsystems Technology (CMST), Intec Department, Ghent University-imec, Belgium \\ ${ }^{c}$ Department of Applied Physics and Photonics, Vrije Universiteit Brussel, Belgium \\ ${ }^{\mathrm{d}}$ Photonics Research Group, Aston University, United Kingdom
}

\begin{abstract}
In traditional electrical sensing applications, multiplexing and interconnecting the different sensing elements is a major challenge. Recently, many optical alternatives have been investigated including optical fiber sensors of which the sensing elements consist of fiber Bragg gratings. Different sensing points can be integrated in one optical fiber solving the interconnection problem and avoiding any electromagnetical interference (EMI). Many new sensing applications also require flexible or stretchable sensing foils which can be attached to or wrapped around irregularly shaped objects such as robot fingers and car bumpers or which can even be applied in biomedical applications where a sensor is fixed on a human body. The use of these optical sensors however always implies the use of a light-source, detectors and electronic circuitry to be coupled and integrated with these sensors. The coupling of these fibers with these light sources and detectors is a critical packaging problem and as it is well-known the costs for packaging, especially with optoelectronic components and fiber alignment issues are huge. The end goal of this embedded sensor is to create a flexible optical sensor integrated with (opto)electronic modules and control circuitry. To obtain this flexibility, one can embed the optical sensors and the driving optoelectronics in a stretchable polymer host material. In this article different embedding techniques for optical fiber sensors are described and characterized. Initial tests based on standard manufacturing processes such as molding and laser structuring are reported as well as a more advanced embedding technique based on soft lithography processing.
\end{abstract}

Keywords: embedding, fiber Bragg grating, molding, optical fiber sensor, photonic skin, polymer, polymer optical fiber

\section{INTRODUCTION}

\subsection{Flexible sensing foil with integrated optoelectronics}

Within different research projects ${ }^{1,2}$, a new paradigm for flexible optical sensors integrated with electronic modules and control circuitry is developed. The many advantages of optical sensors, and mainly optical fiber sensors, make them very attractive for a wide range of applications. The immunity with regard to EMI, the resistance to harsh environments, the high sensitivity and the possibility in parallelizing the readout all make these sensors more useful than their electronic counterparts for automotive applications, aviation, robotics and others.

We are therefore aiming at developing a generic technology that offers an integrated solution to this increasing demand and will mean a breakthrough in the field of optical sensing. The different projects aim at developing a flexible substrate or foil in which the sensing elements can be integrated and in which also the light sources, detectors and electronic circuitry are embedded or integrated on compact signal processing boards. This artificial and flexible optical sensing foil can then be applied to irregular surfaces (e.g. for distributed sensing applications), on moveable surfaces (e.g. in robotics) or can folded into compact modules (for portable devices, automotive, etc). A schematic view of this approach is shown in Figure 1.

*For more information, contact: Bram.VanHoe@elis.ugent.be; phone + 32 (0)9 26453 70; fax + 32 (0)9 2645374 ; http://www.cmst.be

Optical Sensing and Detection, edited by Francis Berghmans, Anna Grazia Mignani, Chris A. van Hoof, Proc. of SPIE Vol. 7726,772603 - @ 2010 SPIE · CCC code: 0277-786X/10/\$18 - doi: 10.1117/12.854865 


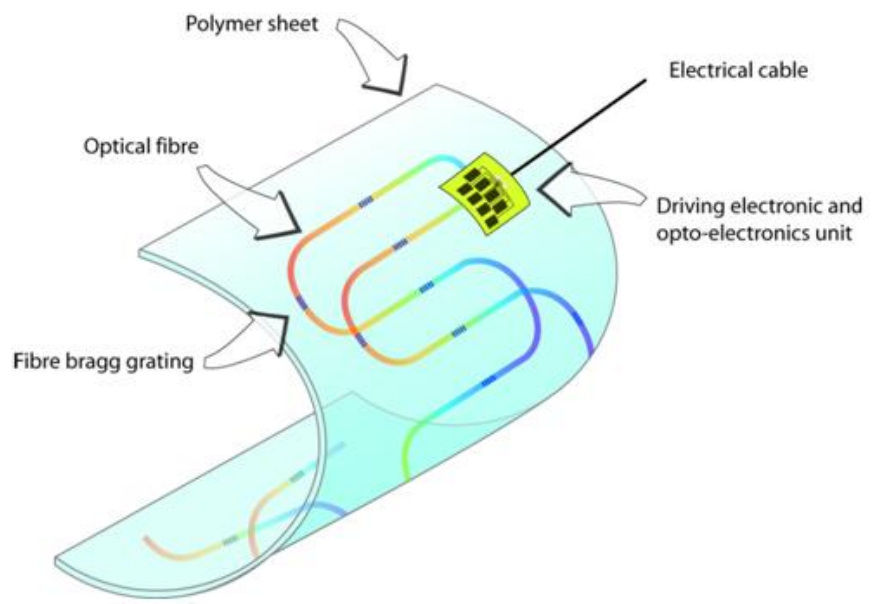

Figure 1: Principle scheme of the embedded fiber sensor with integrated optoelectronic sources and readout unit.

\subsection{Embedding traditional single mode silica fibers}

The embedding of fiber sensors in a host material to measure strain, temperature or pressure has been investigated in different research areas, such as structural health monitoring. Many new applications however require optical sensors embedded in a flexible or even stretchable host material. The resulting sensing sheet, an artificial skin can then be attached to or wrapped around an irregularly shaped object. Fiber Bragg gratings in single mode fibers show a similar sensitivity for different external parameters, such as temperature and strain ${ }^{3}$. The peak reflected wavelength $\Delta \lambda_{B}$ shifts by an amount in response to strain $\varepsilon$ and temperature change $\Delta \mathrm{T}$ as given by

$$
\frac{\Delta \lambda_{B}}{\lambda_{B}}=P_{e} \varepsilon+\left[P_{e}\left(\alpha_{f}+\zeta\right)\right] \Delta T
$$

$\mathrm{P}_{\mathrm{e}}$ is the strain-optic coefficient; $\alpha_{\mathrm{f}}$ is the thermal expansion coefficient of the fiber and $\zeta$ is the thermo-optic coefficient. At a wavelength of $1550 \mathrm{~nm}$, one typically finds following strain and temperature sensitivity for single mode fibers:

$$
\begin{gathered}
\frac{\Delta \lambda_{B}}{\Delta \varepsilon}=1.21 \frac{\mathrm{pm}}{\mu \text { strain }} \\
\frac{\Delta \lambda_{B}}{\Delta T}=10.35 \frac{\mathrm{pm}}{{ }^{\circ} \mathrm{C}}
\end{gathered}
$$

Embedding these fibers in polymer host materials affects the different sensitivities. The embedding of single mode optical fiber Bragg gratings has been investigated using traditional embedding techniques such as molding and laser ablation.

\subsection{Drawbacks and possible improvements}

Both the fibers and the embedding techniques have shown some major drawbacks and optimization research in the type of fiber sensor as well as in the embedding techniques are described in this paper. Possible alternatives for traditional fiber Bragg gratings in single mode silica mode are microstructured silica fibers or polymer optical fibers, both showing compatibility with various fiber Bragg inscription methods. A more advanced embedding technique based on a soft lithography concept has also been investigated. This embedding process starts from an SU-8 master mold which accurately transfers a series of tracks into the flexible host material. Using this technique, an embedding structure can be defined on a micrometer accuracy level. After releasing the host material layer from the mold, fibers can be subsequently aligned and fixed in the patterned tracks. 


\section{DIFFERENT EMBEDDING TECHNIQUES}

Various embedding techniques have been investigated to integrate the optical fiber in a flexible sensing sheet and create a photonic skin. Depending on the needs of the application, one can select a softer or a harder material.

\subsection{Embedding materials}

A thermally curable Polydimethylsiloxane (PDMS) material, Sylgard $\left(184\right.$ from Dow Corning ${ }^{4}$, has been selected as a flexible and stretchable embedding material. This material is a popular embedding material, enabling for example integrated electronics leading to smart textiles ${ }^{5}$. Embedding optical fibers in this stretchable host material is a totally new application however. Some parameters indicating the expected behavior when applying strain or temperature are shown in

Table $1^{6}$.

Table 1. Parameters of Sylgard®184

\begin{tabular}{|l|l|}
\hline \multicolumn{1}{|c|}{ Parameter } & \multicolumn{1}{c|}{ Value } \\
\hline CTE & $310 \mathrm{ppm} /{ }^{\circ} \mathrm{C}$ \\
\hline Young's modulus & $1.2 \mathrm{MPa}$ \\
\hline Poisson coefficient & 0.46 \\
\hline Refractive index & 1.4235 \\
\hline
\end{tabular}

The curing process consists of mixing the silicone product with a curing agent (ratio 10:1). A slow curing process at $60^{\circ} \mathrm{C}$ for 2 hours is applied.

A harder alternative as embedding material is also available, Ormocer ${ }^{\circledR}$ from Microresist ${ }^{7}$. The embedding results using this embedding material are not discussed in this paper.

\subsection{Molding}

The first embedding technique is injection molding using a PMMA mold. In this approach, the fiber is temporarily fixed in channels at the edge of the mold. A slow curing and cooling process is needed in order not to expose too much stress to the embedded fiber caused by the CTE mismatch (difference in CTE is $310 \mathrm{ppm}$ for Sylgard ${ }^{\circledR} 184$ versus 5.5 ppm for a standard silica fiber).

The result of the injection molding process is illustrated in Figure 2.

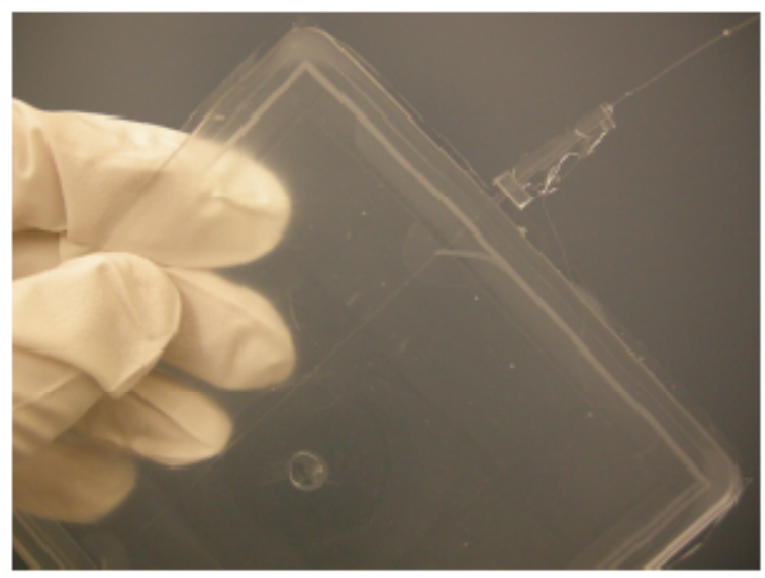

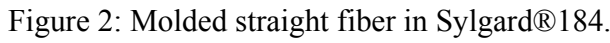


To make not only straight embedded fibers but also other designs (e.g. horse shoe), the necessity of a special designed mold is inevitable. The fiber has then to be fixed not only on the edges of the mold but within the entire mold. A major drawback of this embedding technique is consequently the necessity of a new mold for every new design.

\subsection{Laser ablation}

Laser ablation can be an alternative to easily adapt the fiber embedding design. Starting from a fully cured sheet, a track can be ablated to embed the fiber in. Concerning this laser structuring approach, three different laser sources have been evaluated for this purpose: a $\mathrm{KrF}$ Excimer $(\lambda=248 \mathrm{~nm})$, a frequency tripled Nd-YAG laser $(\lambda=355 \mathrm{~nm})$, and a $\mathrm{CO}_{2}$ laser $(\lambda=10.6 \mu \mathrm{m})$. Due to the small spot size, the Nd-YAG laser was found to be less suited. Both Excimer and $\mathrm{CO}_{2}$ laser could yield in good results, depending on the absorption of the laser wavelength by the flexible foil material. For the Sylgard®184 PDMS material, the $\mathrm{CO}_{2}$ laser provided the best results using a pulse frequency of $100 \mathrm{~Hz}$ and an ablation speed of $8.7 \mathrm{~mm} / \mathrm{s}$ together with minimal power attenuation (maximum pulse energy). After ablating tracks in the silicone, a glue together with a primer to ensure the adhesion of the fiber is needed to fix the fiber within these tracks.

A recent popular design within the research area of stretchable electronics to embed metal tracks in an elastic foil is the use of a horseshoe shape ${ }^{8}$ resulting in a meandering embedding design. This allows non-stretchable materials (such as copper or silica) to be bent or stretched. Minimum radius of the meanders will depend both on the type of fiber and the fiber fixation method (adhesives).

The main advantage of using laser structuring is the ease to make a new design. Any CAD file can easily be exported to a laser drilling file. A result of a fiber embedded into laser structured silicones can be seen in Figure 3.

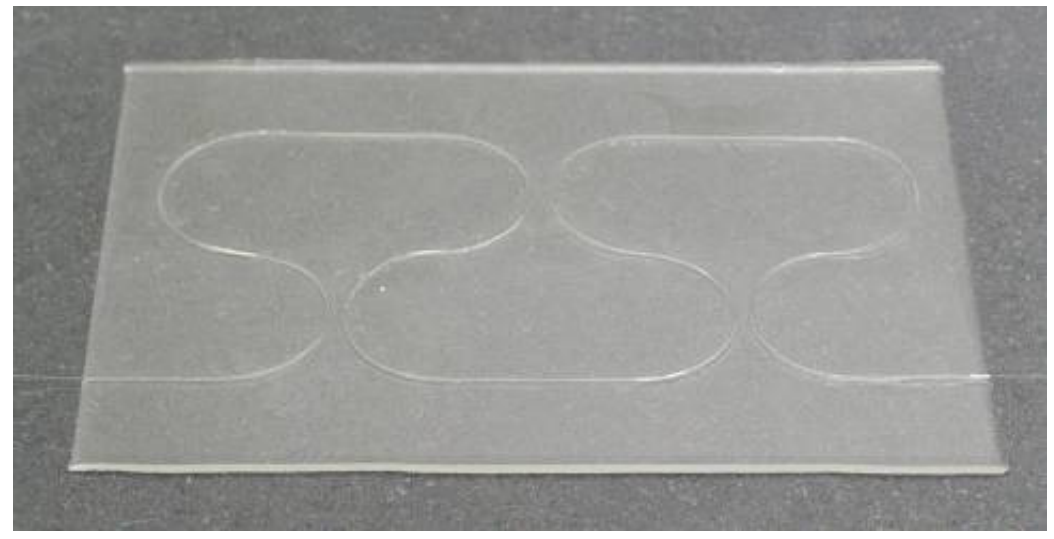

Figure 3: Embedded fiber using laser ablation to define horse shoe shaped structures.

\subsection{Soft lithography approach}

An advanced fiber embedding technique has been investigated to control the fiber position in the optical skin even more accurately. The technique is based on a soft lithography process which essentially consists of the transfer of a polymer master mould in the PDMS host material. As a master mould carrier, we use a silicon wafer but this can be any other substrate which has a low surface roughness. An SU-8 layer is spincoated and patterned by UV contact lithography. This polymer structure will serve as a negative mask for the PDMS material. After a hard bake step of the SU-8, a controlled amount of PDMS material is poured on the silicon wafer and cured at $60^{\circ} \mathrm{C}$. The bad adhesion between the UV patterned polymer and the cured PDMS enables a smooth release of the PDMS material once it is fully cured. The result is a patterned PDMS layer with U-shaped grooves (up to a thickness of $150 \mu \mathrm{m}$ ). This process is illustrated in Figure 4.

The next step is the placing, aligning and fixing of the fiber. Depending on the track width and height, this can be done by either simply pressing the fiber into the tracks or using a UV glue. This last technique has the disadvantage of introducing a hard, non-flexible intermediate layer between the fiber and the host material but will allow a better transfer of the strain/pressure applied on the sensing sheet to the fiber Bragg grating embedded in the flexible host material.

The top layer thickness can be controlled by using a hot embossing press: by controlling embossing pressure and temperature, the fiber embedding tracks can be filled up and the top layer thickness can be adjusted. A liquid (not cured) PDMS layer serves as glue between the bottom layer including the embedded fiber and the (half cured) top layer. 
Next to the accurate fiber positioning, other advantages are the possibility to re-use the SU-8 mould and the convenience to adapt the fiber embedding pattern (also enabling meandering tracks in the PDMS material) by changing the mask design for the UV patterned master mould.

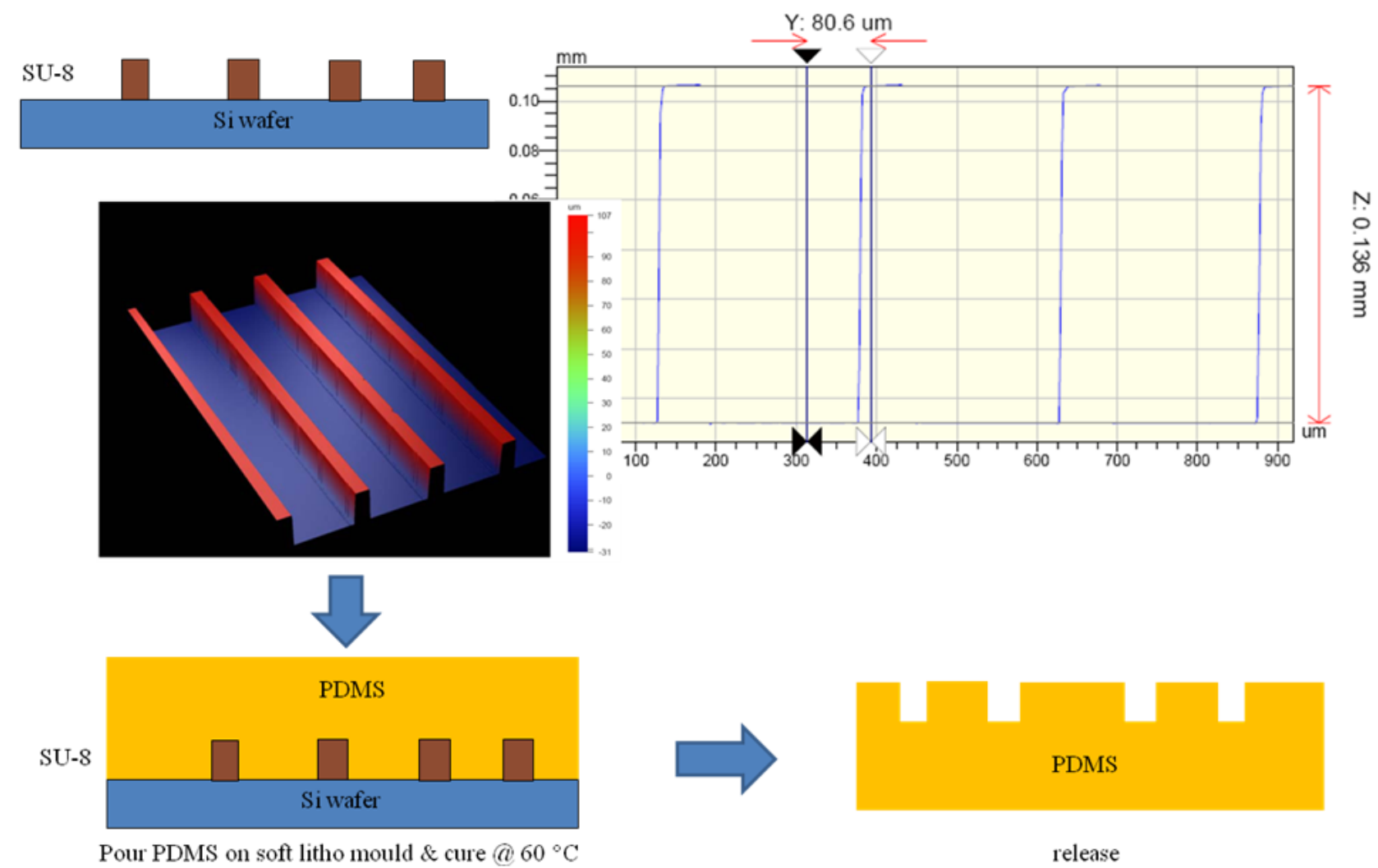

Figure 4: Overview of the transfer of a SU-8 master mould in the PDMS host material.

Two cross section results of an embedded fiber in a PDMS stack are shown in Figure 5 and Figure 6 showing the different embedding layers and the exact fiber position. By adjusting the spinning speeds and the applied pressure during the hot embossing process, one can control the thickness of the different layers.

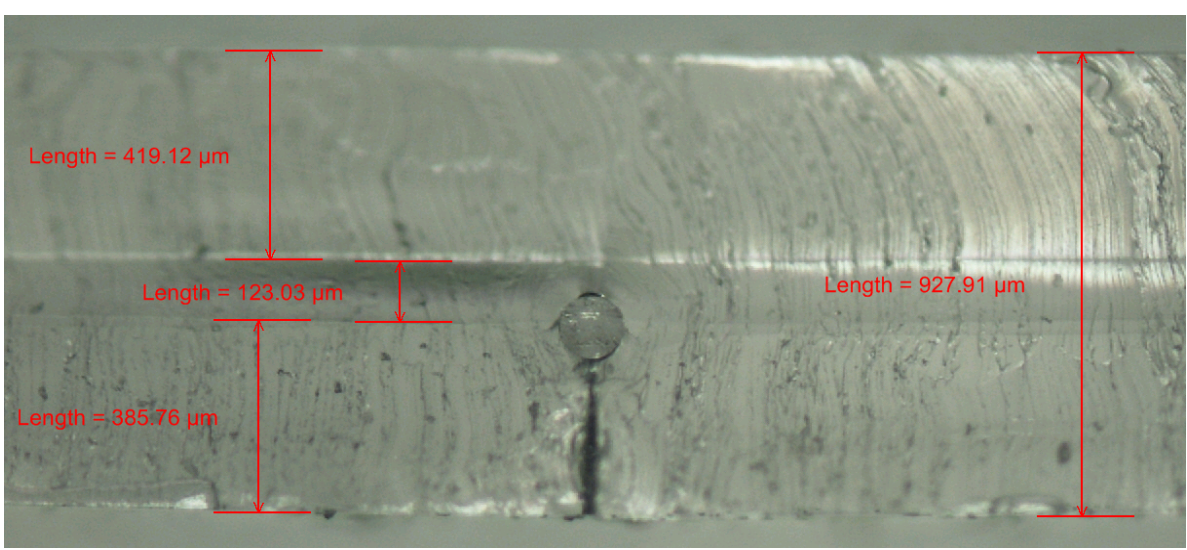

Figure 5: Cross section of an embedded bare silica (total diameter $125 \mu \mathrm{m}$ ). 


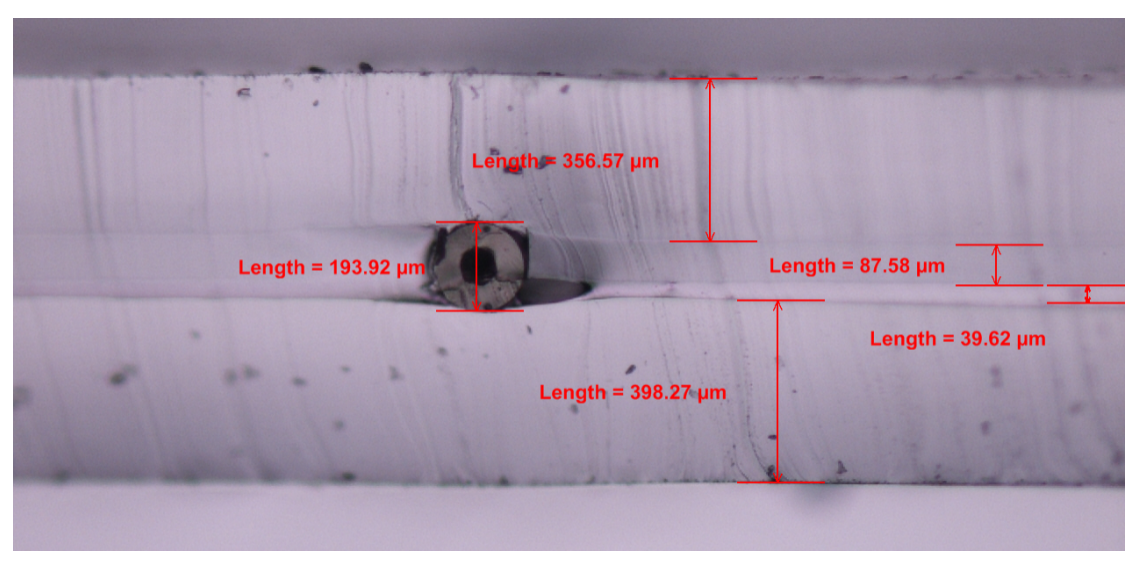

Figure 6: Cross section of an embedded silica fiber with coating (total diameter $190 \mu \mathrm{m}$ ).

\section{CHARACTERIZATION OF EMBEDDED SINGLE MODE OPTICAL FIBERS}

Single mode silica fiber sensors have been embedded using the laser ablation approach, starting from a fully cured sheet. The acrylate fiber coating is locally stripped on the positions of the fiber Bragg gratings. The fiber sensors have a Bragg wavelength in the $1550 \mathrm{~nm}$ range and the embedding material is the commercially available PDMS host material, Sylgard ${ }^{\circledR} 184$ from Dow Corning ${ }^{4}$. Both temperature tests, using a hot plate, and strain tests, using an Instron ${ }^{\circledR}$ strain setup, have been carried out. The results are shown in Figure 7, Figure 8, Figure 9 and Figure 10.

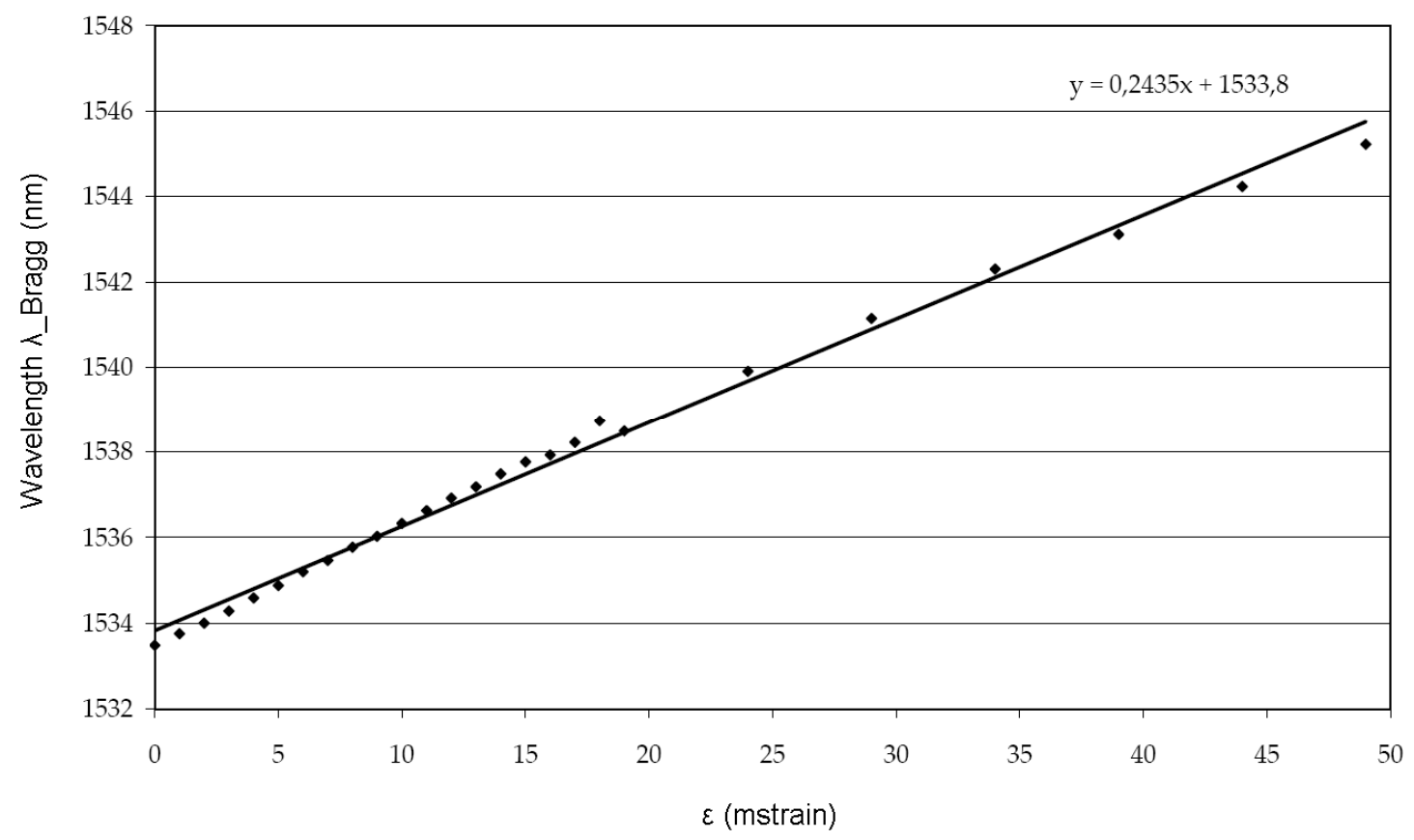

Figure 7: Wavelength shift of the Bragg wavelength versus strain for a straight embedded fiber. Fiber ends are not clamed during the strain test. 


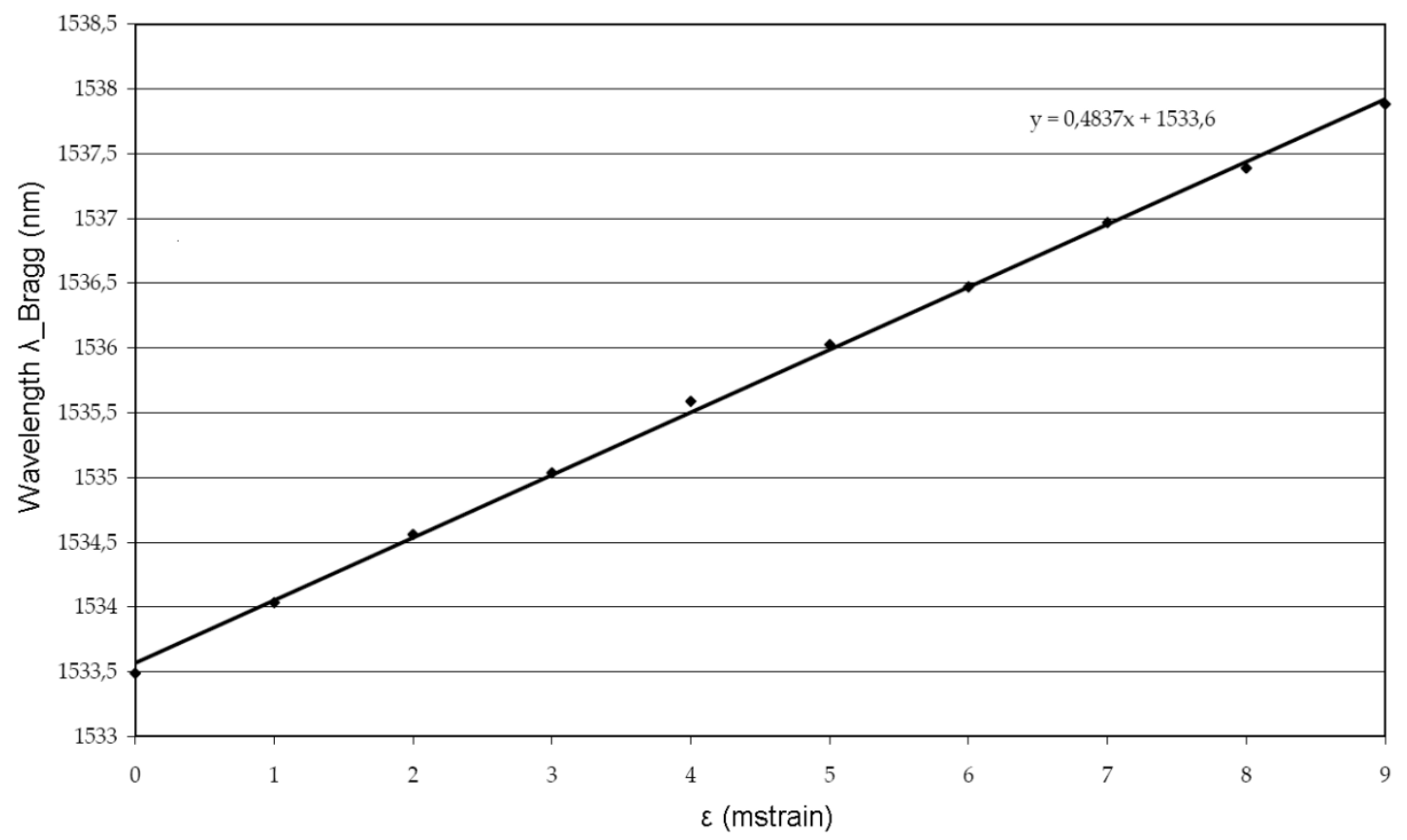

Figure 8: Wavelength shift of the Bragg wavelength versus strain for a straight embedded fiber. Fiber ends are clamed during the strain test.

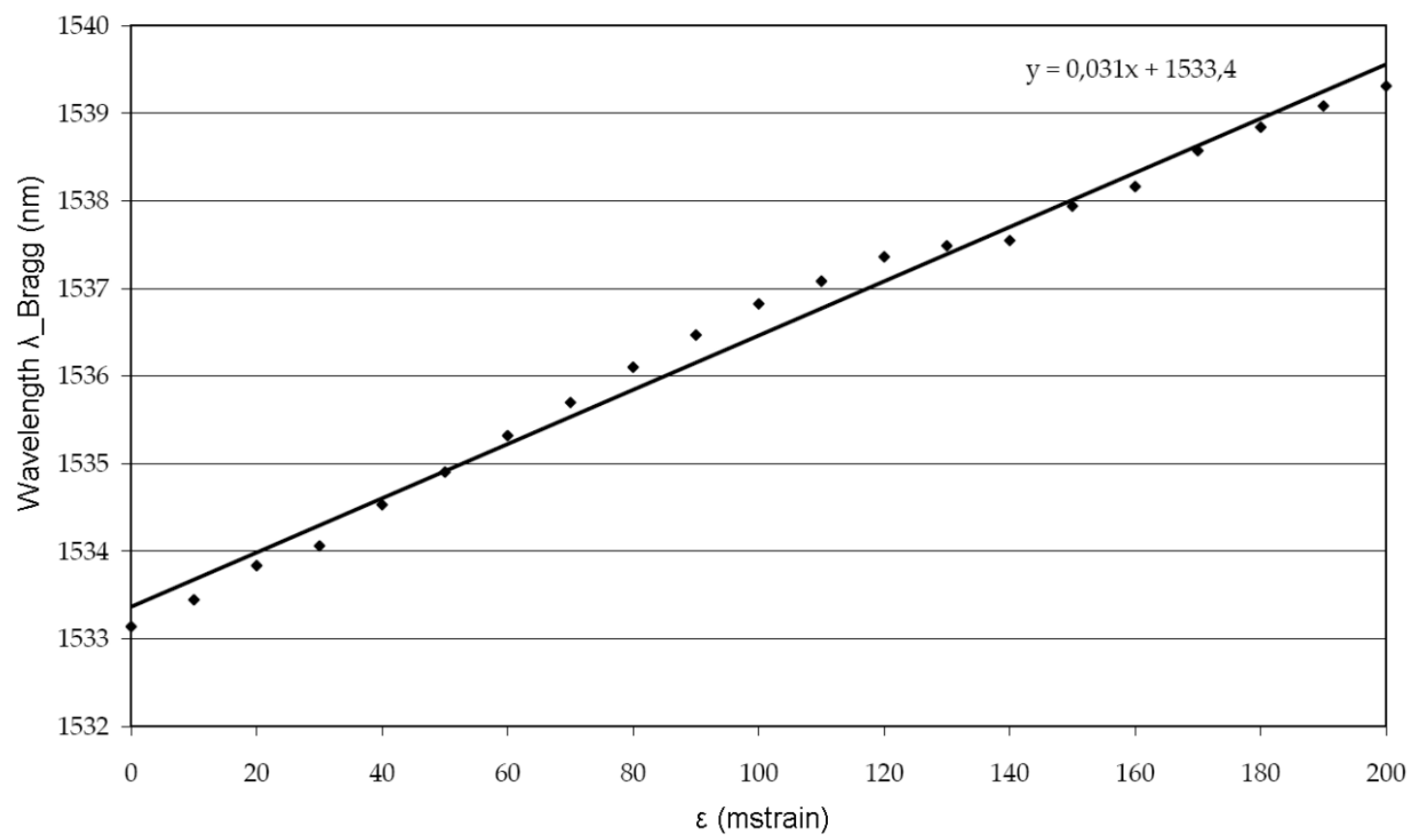

Figure 9: Wavelength shift of the Bragg wavelength versus strain for a straight embedded fiber. Meandering fiber embedding design. 


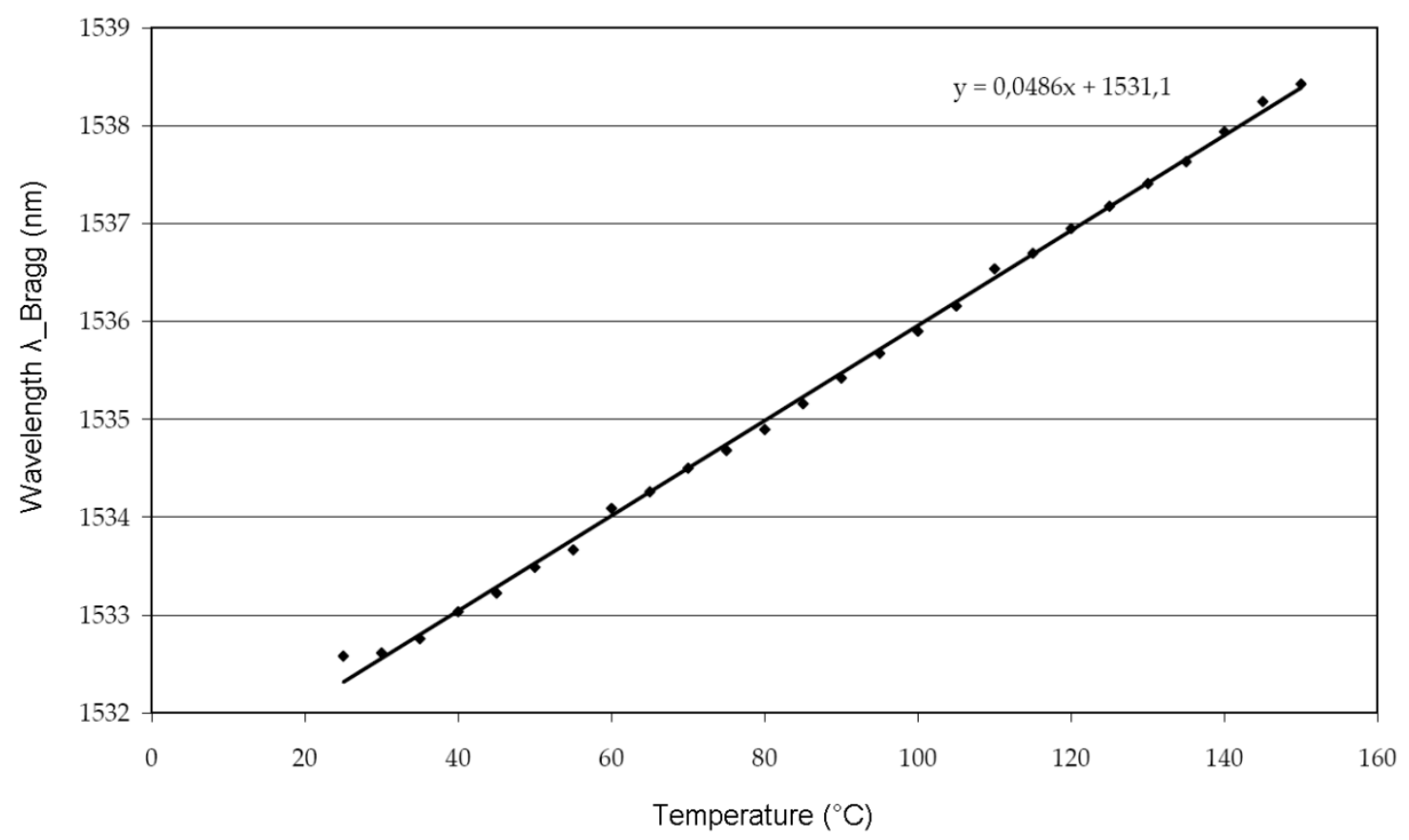

Figure 10: Wavelength shift of the Bragg wavelength versus temperature for a straight embedded fiber.

Figure 7 shows the shift in the Bragg wavelength when strain is applied on a straight embedded fiber. The clamps of the Instron ${ }^{\circledR}$ strain set-up are on the sensor skin and not on the fiber itself. One can deduct a strain sensitivity of $0.24 \mathrm{pm} / \mu$ strain. The difference with the sensitivity of a bare fiber (2) is because of subsequently slipping of the fiber through the fiber coating and of the fiber coating through the silicone sensor skin.

Figure 8 shows the Bragg wavelength shift when clamping both the skin and the fiber. The sensitivity increases to $0.48 \mathrm{pm} / \mu$ strain because of the clamping on the fiber coating. The value mentioned in (2) is not reached however, again because of the slipping of the fiber in the fiber coating. One can conclude that half this sensitivity is due to the clamping of the fiber and, more importantly, half the sensitivity is induced by embedding the fiber in the photonic skin. At the location of the Bragg grating, the sensor embedding material is in direct contact with the bare fiber.

Figure 9 shows the strain sensitivity of one embedded horse shoe shaped fiber design. Because of this meandering structure, the photonic skin will be able to be stretched much further. As a consequence, stretching the foil will not be limited by the fiber stiffness but by the embedding material, when cracks are introduced in the Sylgard embedding material. Because of the meander, the effective strain transfer on the fiber is much less when applying strain with the Instron ${ }^{\circledR}$ set-up. A sensitivity of $0.03 \mathrm{pm} / \mu$ strain is the result.

The influence of temperature on the Bragg wavelength is shown in Figure 10, resulting in a sensitivity of $49 \mathrm{pm} /{ }^{\circ} \mathrm{C}$. Comparing to (3), the sensitivity is multiplied by a factor 5. The reason is the high CTE of the skin material affecting the embedded fiber.

The cross sensitivity to strain and temperature of the single mode fiber sensors is a fundamental problem when measuring transversal strain or pressure. Changing the ambient temperature causes an unwanted shift of the Bragg wavelength which is even enhanced due to the embedding in a flexible foil.

\section{POLYMER OPTICAL FIBERS}

The single mode silica fibers can be replaced by other fibers. A first option is the use of microstructured silica fibers, minimizing the cross sensitivity of the fiber. First trials to embed these fibers in polymer host materials are ongoing and are not discussed in detail here. Another possibility is the use of polymer optical fibers, avoiding the stiff silica material and providing flexibility to the fiber element as such. This will consequently improve the flexibility and stretchability of the embedded sensor. 


\subsection{Fiber Bragg gratings in polymer optical fibers}

This alternative fiber which was embedded in the stretchable host material is a polymer optical fiber (POF). The poly(methyl methacrylate) (PMMA) based POF was a single mode step index fiber, supplied by Prof. Gang Ding Peng of the University of New South Wales, Australia, with a core diameter of $9.5 \mu \mathrm{m}$ and a cladding diameter of $200 \mu \mathrm{m}$. The FBG was inscribed using $30 \mathrm{~mW}$ of continuous wave light at $325 \mathrm{~nm}$ obtained from a HeCd laser (Kimmon model IK5652R-G). With the $7.5 \mathrm{~cm}$ long fiber mounted horizontally in a V-groove, the $1.8 \mathrm{~mm}$ diameter beam was focused vertically down onto the fiber axis using a cylindrical lens of focal length $10 \mathrm{~cm}$. A phase mask with period $1057 \mathrm{~nm}$ was placed on top of the fiber to generate a periodic intensity modulation in the core region. The laser beam was scanned 6 $\mathrm{mm}$ along the phase mask during the 30 minute recording time. For further details of the inscription arrangement, see references ${ }^{9}$.

The inscription process was monitored by butt-coupling the angle-cleaved end of a single mode silica fiber coupler to the POF using a drop of index matching oil, the end of the POF having previously been cleaved using a razor blade on a hot plate at $80^{\circ} \mathrm{C}$. Using the coupler, the POF was illuminated with broadband light covering the range $1530 \mathrm{~nm}$ to $1610 \mathrm{~nm}$ (Thorlabs, Broadband ASE light source) and the reflection spectrum monitored using an optical spectrum analyzer (OSA, HP86142A). A silica reference FBG was made in SMF using a frequency doubled argon ion laser with a 1071.9 $\mathrm{nm}$ period phase mask. The laser beam was scanned along the phase mask once to produce a grating $3 \mathrm{~mm}$ in length.

\subsection{Embedding POF in a polymer host material}

Following the inscription of the POF FBG the fiber was glued to a silica fiber pigtail using UV curable adhesive (Loctite 3525). The reflection spectra of the FBGs were monitored before and after embedding in the skin, with the results being shown in Figure 11. In the case of the silica FBG the embedding process induced a decrease in the Bragg wavelength of $0.8 \mathrm{~nm}$, probably caused by shrinkage of the polymer during the polymerization process. This wavelength shift corresponds to a compressive axial strain of $670 \mu \varepsilon^{10}$. By contrast, the POF FBG displayed a decrease in wavelength of $10.8 \mathrm{~nm}$. This order of magnitude larger value is likely to be the result of two factors. Firstly, due to the much lower value of Young's modulus for PMMA than silica ( $3 \mathrm{GPa}^{11}$ vs. $72 \mathrm{GPa}^{12}$, respectively), any compressive forces provided by the shrinking polymer skin will lead to a greater strain in the POF. Secondly, it has been shown ${ }^{13}$ that when FBGs in POF are taken for the first time above about $50{ }^{\circ} \mathrm{C}$, there can be a large permanent blue shift in the Bragg wavelength associated with a shrinkage of the fiber length as order induced during the drawing process relaxes out. Heat generated during the polymerization of the skin may have been responsible for this effect.

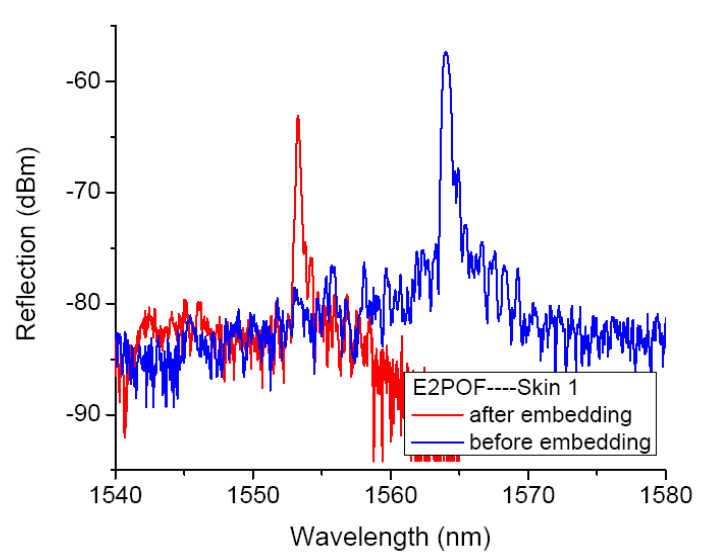

(a)

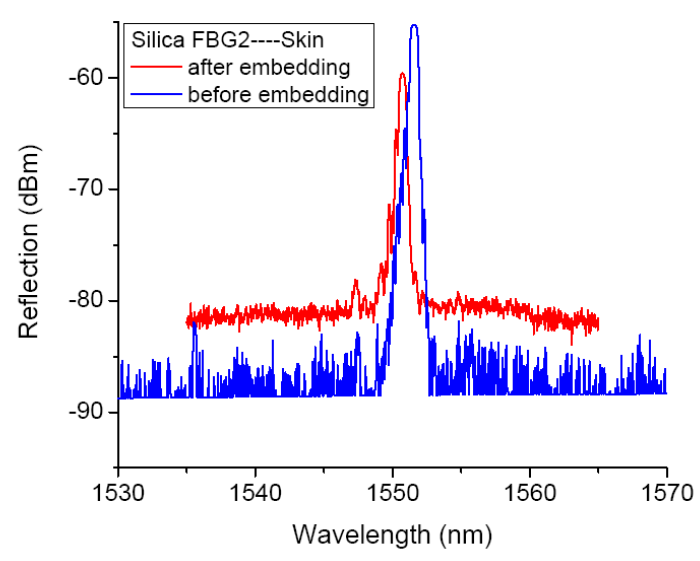

(b)

Figure 11: Reflection spectra of FBGs before (blue line) and after (red line) embedding in the polymer skin.

(a) POF FBG, (b) Silica FBG.

\section{CONCLUSIONS}

The photonic skin which is developed in this paper enables the embedding of optical fiber sensors in flexible and stretchable host materials. As a result, the embedded fiber can be bent, folded and stretched on an irregularly shaped 
object. Adding ambient intelligence to robots or in biomedical applications are the main targets of these embedded sensors.

The sensor elements consist of fiber Bragg gratings written in silica or polymer optical fiber. One of the most important challenges in the fiber and embedding design is coping with the cross sensitivity to various external parameters such as temperature and strain. This sensitivity is also affected by the selected embedding technique and host material. Different fiber embedding techniques have been investigated and a process flow based on soft lithography has shown the best integration principle to control the fiber position in the photonic skin.

Next to the fiber embedding, the integration of the driving optoelectronics and readout elements is also under investigation. The end result will be a fully integrated, flexible and possibly stretchable optical fiber sensor.

\section{ACKNOWLEDGMENTS}

This research is funded by a PhD grant of the Institute for the Promotion of Innovation through Science and Technology in Flanders (IWT-Vlaanderen). This work is also partially conducted within the framework of the project FAOS (Flexible Artificial Optical Skins), which is also supported by the Institute for the Promotion of Innovation through Science and Technology in Flanders (IWT-Vlaanderen), Flanders, Belgium and the EU-FP7 project PHOSFOS (Photonic Skins for Optical Sensing), supported by the European Commission.

\section{REFERENCES}

[1] IWT-SBO project "Flexible Artificial Optical Skin", FAOS, http://intecweb.intec.ugent.be/faos

[2] EU-FP7 project "Photonic Skins for Optical Sensing", Phosfos, http://www.phosfos.eu

[3] Shizhuo Yin, Paul B. Ruffin, Francis T.S. Yu, "Fiber optic sensors", 2008

[4] Dow Corning silicones, http://www.dowcorning.com

[5] F. Bossuyt, T. Vervust, F. Axisa, J. Vanfleteren, "A New Low Cost, Elastic and Conformable Electronics Technology for Soft and Stretchable Electronic Devices by use of a Stretchable Substrate", European Microelectronics and packaging conference (EMPC 2009), vol. 1 and 2, 697-702 (2009)

[6] A. Santiago-Alvarado, S. Vazquez Montiel, J. Munoz-Lopez, J. Castro-Ramos, J. A. Delgado Atencio, "Parametric and Scattering Characterization of PDMS Membranes for Optical Applications”, Proc. SPIE, 7426, 742615 (2009)

[7] Microresist Ormocer ${ }^{\circledR}, \underline{\text { http://www.microresist.com }}$

[8] M. Gonzalez, F. Axisa, M. Vanden Bulcke, D. Brosteaux, B. Vandevelde, J. Vanfleteren, "Design of metal interconnects for stretchable electronic circuits", Microelectronics reliability, 48 (6), 825-832 (2008)

[9] H. Dobb, D.J. Webb, K. Kalli, A. Argyros. M.C.J. Large, M. A. Eikelenborg, "Continuous wave ultraviolet lightinduced fiber Bragg gratings in few- and single-mode microstructured polymer optical fibers", Opt. Lett. 30, 3296-3298 (2005)

[10] A. D. Kersey, M. A. Davis, H. J. Patrick, M. LeBlanc, K. P. Koo, C. G. Askins, M. A. Putnam, E. Joseph Friebele, "Fiber grating sensors", Journal of Lightwave Technology, 15(8), 1442-1463 (1997)

[11] Kiesel S, Peters K, Hassan T, Kowalsky M., "Behaviour of intrinsic polymer optical fiber sensor for large-strain applications", Meas. Sci. Technol., 18(10), 3144-54 (2007)

[12] Kaye GWC, Laby TH., "Tables of Physical and Chemical Constants and Some Mathematical Functions", Longman SC \& Tech (1995)

[13] Carroll KE, Zhang C, Webb DJ, Kalli K, Argyros A, Large MCJ, "Thermal response of Bragg gratings in PMMA microstructured optical fibers", Opt. Express 15(14), 8844-8850 (2007) 